\title{
Soap-free emulsion polymerization of poly (methyl methacrylate-co-butyl acrylate): effects of anionic comonomers and methanol on the different characteristics of the latexes
}

\author{
Hossein Adelnia $\cdot$ Saeed Pourmahdian
}

Received: 9 June 2013 / Revised: 27 July 2013 / Accepted: 27 July 2013 / Published online: 13 September 2013

(C) The Author(s) 2013. This article is published with open access at Springerlink.com

\begin{abstract}
Soap-free emulsion polymerization (SFEP) of methyl methacrylate, butyl acrylate was conducted in water/ methanol media with sodium salts of four different acidic comonomers, namely styrene sulfonic acid (NaSS), 2acrylamide-2-methyl-1-propane sulfonic acid (NaAmps), acrylic acid (NaAA), and itaconic acid $\left(\mathrm{Na}_{2}\right.$ ita). It was found that the introduction of methanol as co-solvent ( $35 \mathrm{wt} \%$ ) to the medium greatly decreases the amount of water-soluble polyelectrolyte in the cases $\mathrm{NaAA}$ and $\mathrm{Na}_{2}$ ita while it does not make difference for NaSS and NaAmps. Having employed the concept of conductance dependency to the ion mobility, the onset concentration in which soluble chains were formed was detected. The addition of sulfonic-based comonomers (NaSS and NaAmps), first decreased particle size and then led to predomination of solution polymerization over SFEP. On the contrary, the incorporation of carboxylic-based comonomers ( $\mathrm{NaAA}$ and $\mathrm{Na}_{2} \mathrm{ita}$ ) led to increase in particle size. Moreover, the particle size results were in good qualitative agreement with the classical Smith-Ewart theory.
\end{abstract}

Keywords Soap-free emulsion polymerization · Particles · Anionic functionalized $\cdot$ Comonomer

\section{Introduction}

Synthetic polymeric latexes have recently attracted the attention of both scientific and industrial communities all around the world. These latexes have been used not only in numerous

H. Adelnia $\cdot$ S. Pourmahdian $(\bowtie)$

Polymer Engineering and Colour Technology Department,

Amirkabir University of Technology, Tehran, Iran

e-mail: pourmahd@aut.ac.ir traditional applications [1] but also in state-of-the-art areas like solar cells [2], proton-conducting membranes [3], drug delivery systems [4], and so on. Since the presence of emulsifier or stabilizer in these latexes is associated with several disadvantages $[5,6]$, deep attention has been paid to the techniques in which stable dispersions achieve without using any stabilizer or surfactant.

Soap-free emulsion polymerization (SFEP) is one of them in which stability of the latex is commonly achieved by electrical charges induced by ionic groups arising from initiator and comonomer. Ionic initiator alone cannot suitably endow dispersion stability, surface charge density, and particle size reduction unless large amount of that is employed which consequently leads to low molecular weight; thereby, the incorporation of ionic comonomer is essential in order to fulfill those requirements. Various comonomers such as (meth) acrylic acid, itaconic acid [7], sodium salt of styrene sulfonic acid (NaSS) [8], dimethylaminomethacrylate methyl chloride [9], [2-(methacryloyloxy)ethyl]trimethyl ammonium chloride [10], oleic acid [11], and etc. have so far been utilized in SFEP to control particle size, and its distribution. It is wellunderstood that the introduction of comonomer intensifies electrostatic repulsion, resulting in higher colloidal stability along with lower final particle size. Nevertheless, in some cases, it either leads to increased particle size, broad size distribution, destabilization, or the formation of free polyelectrolyte chains [10].

SFEP has traditionally been carried out in water as the medium. However, some researchers have recently examined the addition of a water miscible co-solvent to the medium. This dramatically influences the nucleation stage which commonly takes place in the continuous phase. On one hand, the addition of co-solvent can enhance solubility of growing chains and delay the precipitation of them, leading to fewer 
numbers of nuclei. One the other hand, it can facilitate the monomer diffusion (i.e., increased solubility of monomer in the medium), resulting in higher number of nuclei. The consequences of the former and the latter are increase and decrease in particle size, respectively. Regardless of these points, the existence of monomer droplets in SFEP is accompanied by some difficulties such as sensitivity of particle size and particle size distribution on the agitation speed [12, 13], and chemical chain heterogeneity in production of copolymers [14]. Utilizing ethanol as co-solvent, Lio et al. [10] restricted the formation of free polyelectrolyte chains and adjusted particle size. Chonde et al. [15] reported that the addition of methanol in SFEP of styrene narrows particle size distribution. An et al. [16] also demonstrated the efficiency of acetone as co-solvent in SFEP of crosslinked PMMA. Despite great efforts, there are some controversies both in restricting the formation of free polyelectrolyte by co-solvent and adjusting particle size by comonomers, especially anionic ones.

Therefore, this work focuses on three main objects including: first, investigating the effect of methanol as a co-solvent to restrict the formation of free polyelectrolyte chain; second, examining four different anionic comonomers to prepare stable poly(methyl methacrylate-co-butyl acrylate) latexes; and finally, interpreting the results and comparing them with each other as well as previous papers in terms of the average particle size, particle size distribution, and stability.

\section{Materials and methods}

\section{Materials}

Methyl methacrylate (MMA), butyl acrylate (BuA), acrylic acid (AA), itaconic acid (Ita), ammonium persulfate (APS), sodium hydroxide $(\mathrm{NaOH})$, and methanol were purchased from Merck Co. NaSS were received from Sigma-Aldrich Co. AA and Ita were neutralized by equivalent amount of $\mathrm{NaOH}$ solution to prepare $\mathrm{NaAA}$ and $\mathrm{Na}_{2}$ ita, respectively. All of the materials were used without further purification.

Typical stabilizer-free dispersion polymerization

MMA/BuA $(6.8 / 1 w / w)$ mixture were used as stock. In order to synthesize MMA-BA copolymer (MBC)-based particles, typical SFEP was carried out as follows: $19.76 \mathrm{~g}$ water was added to $10.64 \mathrm{~g}$ methanol as polymerization medium. Monomer phase, $1.6 \mathrm{~g}$ in total including $0.016 \mathrm{~g}$ APS $(1 \mathrm{wt} \%$ with respect to total monomer), was made of $1.52 \mathrm{~g}$ of MMA/BuA mixture and $0.08 \mathrm{~g}$ of NaSS ( $2.5 \mathrm{~mol} \%$ with respect to total) which were added to the medium. The ingredients of the recipe (mixture of methanol/water and monomer phase) were added to 50-cc glass bottles, purged with nitrogen, capped and sealed. Each glass bottle was located in a constant-temperature water bath at $75^{\circ} \mathrm{C}$ at $60 \mathrm{rpm}$. The reaction times for all runs were about $24 \mathrm{~h}$ to assure a complete reaction.

\section{Characterization}

A small amount of a latex dispersion was diluted with the medium until the solution was almost transparent. A drop of dispersion was placed onto a glass substrate and left to dry. The samples were sputter coated with gold. Dried colloids were examined with a High Resolution LEO 1550 FEF scanning electron microscope (SEM) at acceleration voltage of $10 \mathrm{kV}$.

The average hydrodynamic diameters and polydispersity indexes (PDI) of MBC-based particles were determined by dynamic light scattering (DLS, Nano ZS, Malvern Instruments). DLS was applied with an angle of 173 by using HeNe laser (4 mW) operated at $633 \mathrm{~nm}$.

Conductivities of dispersions were measured using precision conductivity measuring equipment from LKB (Sweden) at $25^{\circ} \mathrm{C}$, the flow conductivity cell 5311 with a cell constant of $0.73 \mathrm{~cm}^{-1}$ at $25^{\circ} \mathrm{C}$, and conductolyzer $5300 \mathrm{~B}$ with a bridge frequency of $2 \mathrm{kHz}$.

The conversion of monomers was determined by gravimetry. Prepared dispersions dried under vacuum at $60^{\circ} \mathrm{C}$ for evaporation of volatile unreacted monomers and medium, then dried samples were immersed in medium. Polymers were completely removed from unreacted comonomer and initiator by performing of centrifugation $(20,000 \mathrm{rpm} / 15 \mathrm{~min}) /$ redispersion three times. The amount of free polyelectrolyte was determined by centrifugation $(20,000 \mathrm{rpm} / 60 \mathrm{~min})$ and gravimetric method. Note that all sample conversions were higher than $95 \%$.

\section{Results and discussion}

Formation of soluble chain in the medium was examined in various methanol contents ranging from 0 to $50 \mathrm{wt} \%$ when $\mathrm{NaAA}, \mathrm{Na}_{2}$ ita, NaSS, and NaAmps were kept constant at $5 \mathrm{~mol} \%$ with respect the total monomer (Fig. 1). All of the samples were stable and free of any coagulum. The amount of the water-soluble polyelectrolyte in the case of carboxylic functionalized particles ( $\mathrm{NaAA}$ and $\mathrm{Na}_{2}$ ita) dramatically decreases as the amount of methanol increases, whereas no noticeable change can be seen in the case of sulfonic functionalized particles (NaSS and NaAmps). These are probably attributed to the non-solvency and solvency of methanol toward homo-polymers of NaAA and NaSS, respectively. So, based on these results, it can be deduced that co-solvents do not necessarily reduce the amount of the soluble polyelectrolyte and it severely depends on the tendency of the polymer to be dissolved in the given medium. 


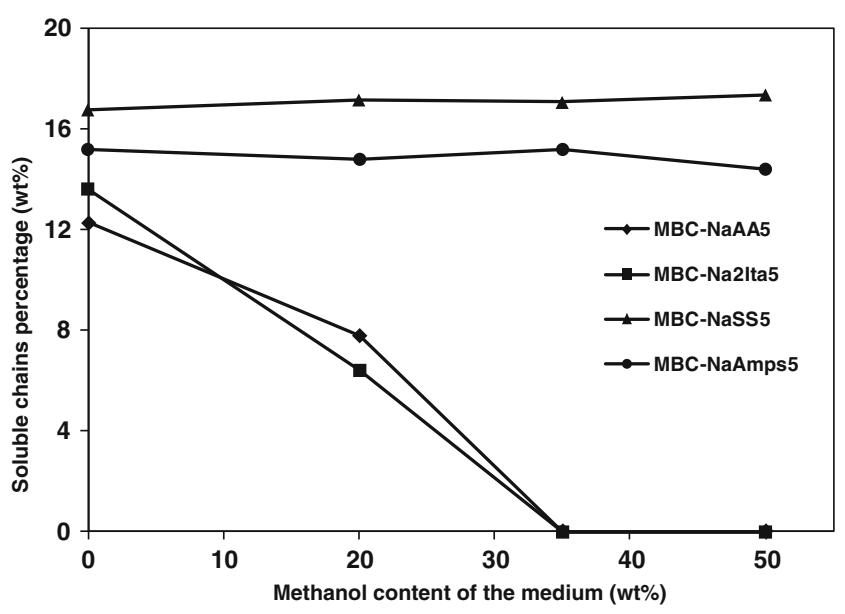

Fig. 1 Amount of soluble polyelectrolyte versus methanol content of the medium in latexes containing $5 \mathrm{~mol} \%$ of different ionic comonomers

Electrostatic charges arising from ionic species along the polymer backbone is responsible for the formation of these soluble chains. This makes the adsorption of growing chains too difficult because of its repulsion by the nuclei; accordingly they cannot easily be captured by preformed nuclei (Fig. 2a). The electrostatic charges also repel adjacent anionic units along a single chain, resulting in the formation of an extended molecular conformation which consequently leads to the solubilization (Fig. 2b). Both of these phenomena lead to the formation of ionic comonomer-rich chains in the medium.

After all, we decided to conduct other reactions in water/ methanol $(65 / 35 w / w)$ not only due to minimization of the free polyelectrolyte chains especially in the case of carboxylic functionalized latexes but also due to elimination of the monomer droplet and achieving homogenous mixture of the reagents at the early stage of the polymerization. The latter case abates sensitivity of particle size and its distribution to the agitation speed, and makes monomers distribution along the copolymer chains more uniform as it was mentioned above. Despite these advantages, as the co-solvent content increases, dielectric constant decreases. Since the thickness of electrical double layer is directly proportional to the dielectric constant, high amount of co-solvent can lead to destabilization. Another

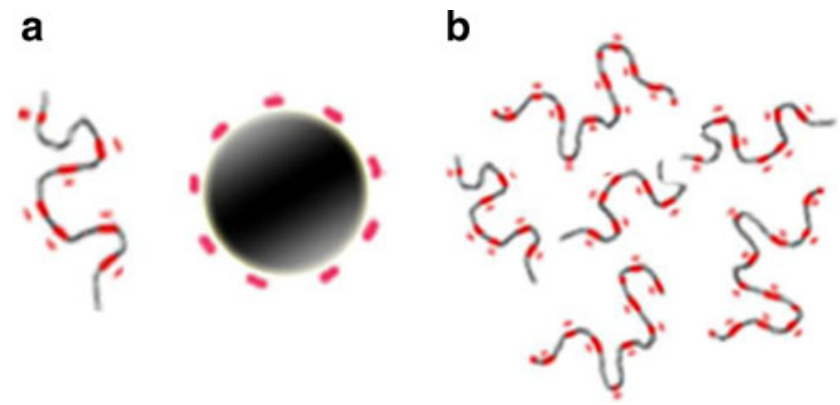

Fig. 2 Schematic representing a repulsion of growing chains by the nuclei, $\mathbf{b}$ extended chain conformation induced by electrostatic repulsion of neighboring units possible influence of co-solvent on the resultant particle size can be induced by the medium viscosity. The nucleation mechanism can be greatly influenced by the viscosity [17]. An increase in viscosity facilitates coagulating of primary particles, and less number of nuclei would be formed at the early stage of the reaction, thereby, an increase in particle size could be expected. Therefore, high amount of methanol should be avoided since it increases the medium viscosity. Based on the abovementioned discussion, the amount of cosolvent must carefully be determined.

Effect of NaSS as comonomer with different concentration

In this set of experiments, NaSS was used with various concentrations to study its effect on different aspect of SFEP and characteristics of final latexes (Table 1).

Figure 3a-f shows SEM images of final MBC with increase in NaSS amount ( $0-5 \mathrm{~mol} \%)$ while using the water/ methanol mixture $(65 / 35, w / w)$ as medium according to the previous section. As it can be seen, the incorporation of NaSS dramatically reduced particle size while a slight increase in particle size distribution is observed (Table 1). However, further increase in NaSS led to partial dissolution of polymer. This dissolution was to such an extent that MBC-based particles with $12.5 \mathrm{~mol} \%$ completely dissolved in the medium.

Even in the absence of any comonomer, anionic sulfate groups of initiator molecules incorporated in the polymer chains endow the MBC-based particles with the colloidal stability (Fig. 3a). In fact, $35 \mathrm{wt} \%$ of methanol as co-solvent did not adversely affect on stability as compared to other studies performed on SFEP in water, without comonomer [17]. Significant particle size reduction, at extremely low content of NaSS, is attributed to the high electrostatic repulsion between nuclei which originated from sulfonic groups of $\mathrm{NaSS}$; as a consequence, the rate of coagulative nucleation reduces which finally leads to higher numbers of nuclei and lower particles size [18]. Subsequent increase in NaSS content further decreased the particle size, but in the lower rate (i.e., exponential behavior of particle diameters versus [NaSS]). These results are in good accordance with the findings of Shi et al. [8], although they used water alone as the medium and kept ionic strength constant. This can signify the fact that even in the presence of $35 \mathrm{wt} \%$ methanol, NaSS is efficient enough to not only keep synthesized latexes stable but also reduce particle size.

Note that since we did not add any inert salt to equalize the ionic strength in these set of experiments, it reasonably increases by the addition of co-monomer; thereby, one can consider that there is a competition between ionic strength and comonomer concentration in which the former contributes to stabilizing nuclei, preventing homo-coagulation, and lowering particle size, while the latter functions against by reducing the thickness of electrical double layer (screening effect). 
Table 1 Effect of NaSS mole percent on different characteristics of MBC-based samples

\begin{tabular}{lllllll}
\hline Sample code & NaSS in feed (mol\%) & particle size $(\mathrm{nm})$ & PDI & Conversion at 15 min & Conversion at 25 min & Final conversion (after 24 h) \\
\hline MBC0 & 0 & 350 & 0.009 & 15.2 & 23.7 & 98.5 \\
MBC-NaSS0.625 & 0.625 & 152 & 0.021 & 17.7 & 45.8 & 99.3 \\
MBC-NaSS1.25 & 1.25 & 147 & 0.035 & 22.9 & 53.6 & 98.4 \\
MBC-NaSS2.5 & 2.5 & 106 & 0.03 & 27.5 & 42.1 & 98.8 \\
MBC-NaSS5 & 5 & 67 & 0.078 & 16.3 & 27.6 & 97.6 \\
MBC-NaSS10 & 10 & - & - & 9.1 & 18.5 & 99.9 \\
\hline
\end{tabular}

Considering these facts, it can be deduced that comonomer concentration has overcome the ionic strength.

Irrespective of what were discussed, there is another important phenomenon concerning the introduction of comonomer: in the polymerization methods in which the homogenous nucleation governs (like traditional emulsion and SFEP), the final particle size strongly depends on the critical oligoradical length above which they precipitate to form primary particles [19]. The incorporation of the hydrophilic comonomers increases the critical precipitation length of growing chains; as a result, less number of nuclei precipitates and the particle size increases. If this phenomenon had predominantly occurred in the case of $\mathrm{NaSS}$, the particle size and the conversion at the early stage of the polymerization should respectively have been increased and decreased, while they did not (Table 1). Taking these facts into account, it can be concluded that this effect had been negligible herein. This is likely due to reactivity ratios difference. Regardless of the effect of $\mathrm{BuA}$ on the reaction (due to its low content with respect to the MMA), the free radical copolymerization reactivity ratios for $\mathrm{MMA} / \mathrm{NaSS}$
Fig. 3 SEM micrographs of MBC-based particles synthesized by SFEP at $75^{\circ} \mathrm{C}$ using water/ methanol $(65 / 35 \mathrm{w} / \mathrm{w})$ as the polymerization medium with various molar percentage of NaSS with respect to total monomers: 0 (a), 0.375 (b), 0.75 (c), 1.25 (d), 2.5 (e), and 5 (f)
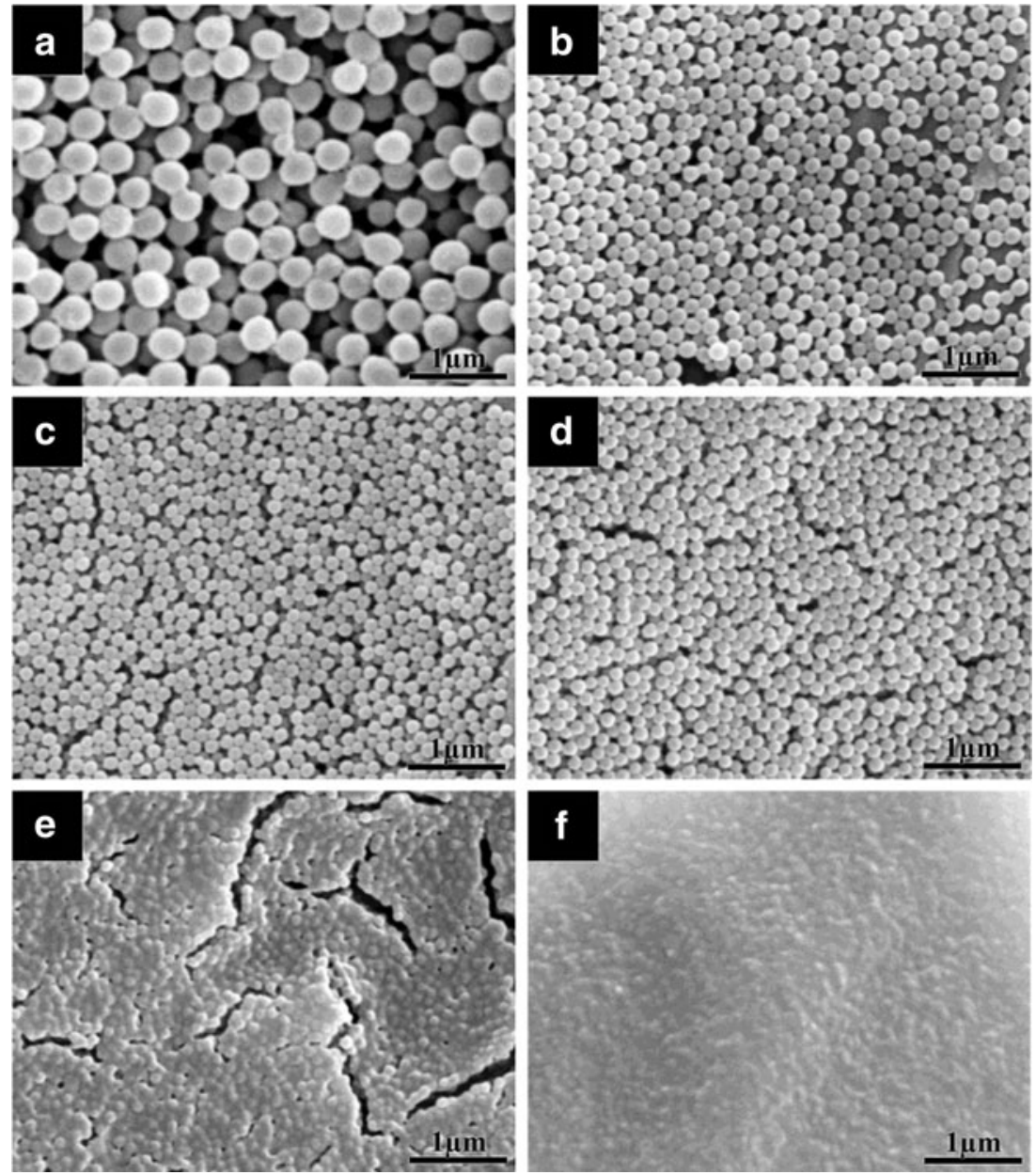
system are $r_{\mathrm{MMA}}=1.13$ and $r_{\mathrm{NaSS}}=0.52$ [20]. Lower reactivity of NaSS as compared to the MMA causes less NaSS units to incorporate in the chain at the early stage of the polymerization.

To get a better understanding of the solubility, we performed conductometry test. The results in Fig. 4 show that the conductivity has increased with NaSS mole fraction. A deeper observation over the evolution of conductivity reveals a break point which seems to be the onset of partial dissolution of the polymer. Least square data fitting with a first-order polynominal was done on the results and two different regimes were detected as below (Eqs. 1 and 2):

$$
\begin{aligned}
& G=705+88.6 \times C_{\text {NaSS }}, \quad r^{2}=0.998\left(\text { for } C_{\text {NaSS }} \geq 1.52\right) \\
& G=658+119.1 \times C_{\text {NaSS }}, \quad r^{2}=0.996\left(\text { for } C_{\text {NaSS }} \leq 1.52\right)
\end{aligned}
$$

$G, C_{N a S S}$, and $r^{2}$, respectively, represent the dispersion conductance, the concentration of NaSS in the monomer phase, and linear least square correlation coefficient. Accordingly, Fig. 4 shows two different lines fitted over the results, which crosses each other in $1.52 \mathrm{~mol} \%$ of NaSS. This concentration can be roughly considered as the point in which soluble polyelectrolyte chain form. Generally, conductivity of a given solution can be written in terms of the mobility of all the ions present (Eq. 3) [21].

$$
G=F^{2} \sum_{i} z_{i}^{2} c_{i} u_{i}
$$

In this equation, $F$ is the Faraday constant and the summation covers all ionic species. $z_{i}, c_{i}$, and $u_{i}$ are charge, local concentration, and mobility of $i$ th ionic species, respectively.
Mobility of NaSS units incorporated in the polymer chain depends on its location: buried inside the particles, located on the surface of particles, or dissolved in medium. Obviously, the mobility of those which are soluble in the medium is much higher than those which are located inside or on the particle surface. Considering the dependency of conductance to the ion mobility, Landfester et al. [22] evaluate the stability of miniemulsions. In the same way, Ciszkowska [23] detected coil-to-helix transition temperature of anionic polysaccharides; therefore, based on these studies, the improved conductance of the medium is attributed to the higher mobility and dissolution of terpolymer chains in the medium.

Tendency of the polymers for dissolution at relatively high amount of NaSS is attributed to the high electrical charges induced by NaSS units statically incorporated in the polymer chain as it was for the formation of soluble polyelectrolyte. These charges are also responsible for rod-like conformation of polyelectrolytes [24] and dissolution of hydrophobically alkali soluble emulsions [25]. Moreover, the affinity of MMAbased polymers to alcohols improves the dissolution of the terpolymer in such medium [26].

\section{Effect of NaAmps as comonomer with different concentration}

In this set of experiment, sodium salt of 2-acrylamide-2-methyl1-propane sulfonic acid (NaAmps) was used as comonomer to examine its effect on particle size, and particle size distribution. Other polymerization parameters were kept constant. Figure 5 and Table 2, respectively, show SEM micrographs and different characteristics of MBC-based particles with varying NaAmps concentration.

All of dispersions were stable, even when soluble polyelectrolyte chain formed in $5 \mathrm{~mol} \%$ of NaAmps similar to NaSS
Fig. 4 Latex conductance of NaSS stabilized MBC-based copolymer with different $\mathrm{NaSS}$ concentration. Inset indicates the magnification of the main graph in NaSS concentration range in which break point detect. Blue and red dash lines are the fitted line for $\mathrm{NaSS} \leq 1.52$ and $\mathrm{NaSS} \geq 1.52$, respectively

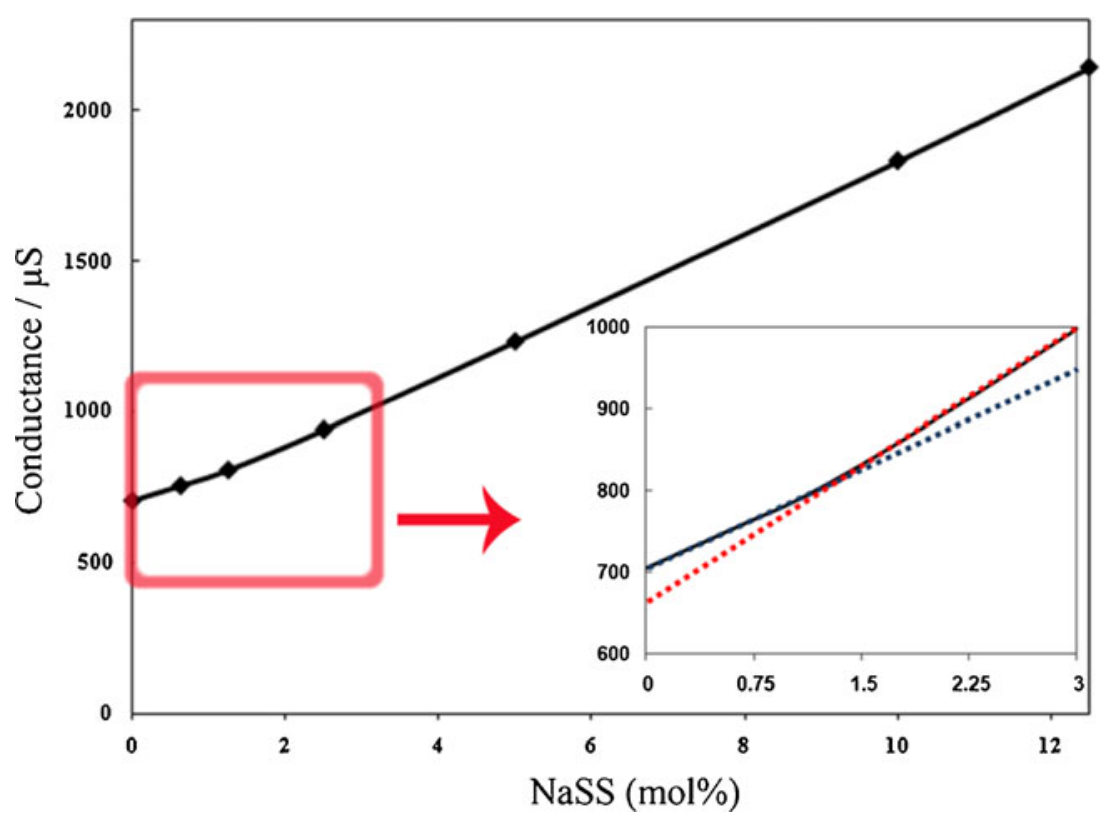


Fig. 5 SEM micrographs of MBC-based particles synthesized by SFEP at $75{ }^{\circ} \mathrm{C}$ using water/ methanol $(65 / 35 \mathrm{w} / \mathrm{w})$ as the polymerization medium with various molar percentage of NaAmps with respect to total monomers: 1.25 (a), $2.5(\mathbf{b}), 5(\mathbf{c})$ and $10(\mathbf{d})$
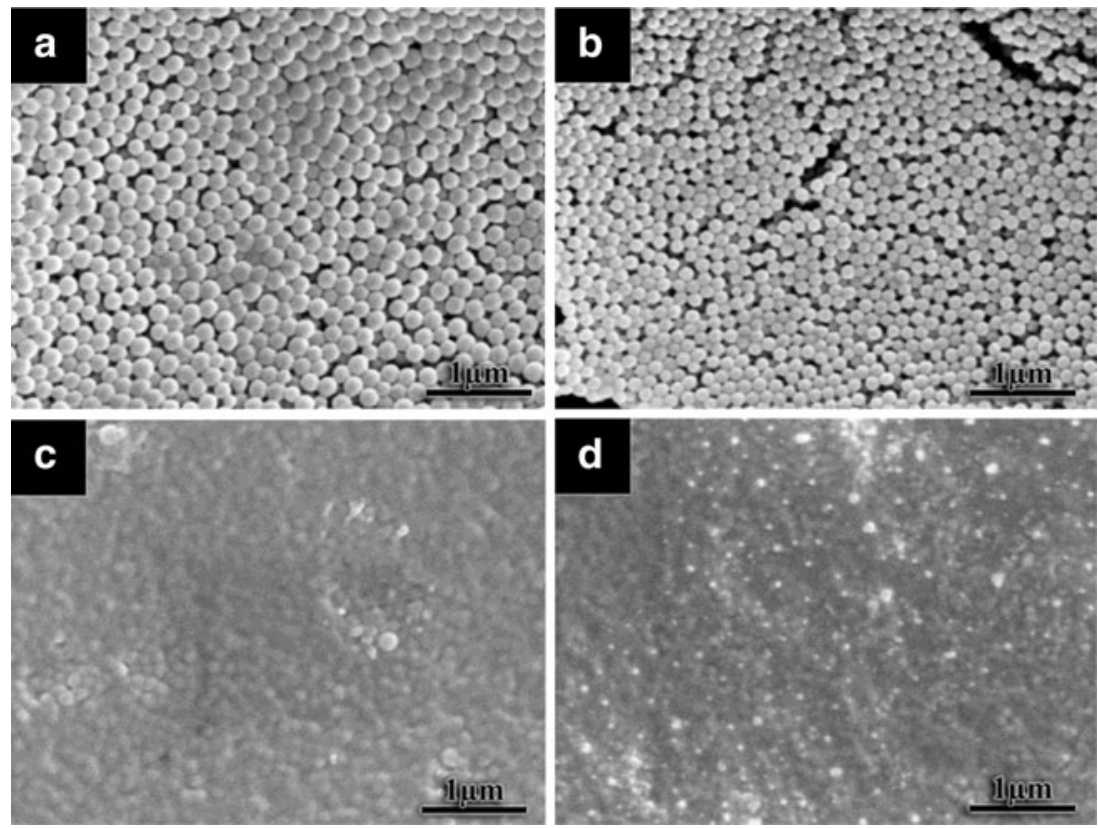

case. Additionally, as the amount of NaAmps increased particle size decreased. Both the results and discussion are similar to $\mathrm{NaSS}$ case. However, as can be seen, at a constant co-monomer concentration, particle size is a little higher as compared to the $\mathrm{NaSS}$ case. Moreover, the fitting of dispersion conductance results revealed the break point to be equal to $1.63 \mathrm{~mol} \%$ of NaAmps which is more than that of NaSS. These results imply that the efficiency of $\mathrm{NaAmps}$ is slightly lower than NaSS.

The rate of polymerization at the initial stages (conversion at $15 \mathrm{~min}$ ), in both cases, first increased and then decreased (Tables 1 and 2). The classical Smith-Ewart theory explains that the rate of polymerization is directly proportional to the number of particles [27]. So, this theory further confirms particle size reduction in relatively low amount of $\mathrm{NaSS}$ and NaAmps. However, at higher amount of these functional comonomers, the conversion decreases. This is due to predomination of solution polymerization route over SFEP. It should be noted that the rate of solution polymerization is generally much slower than conventional emulsion polymerization and SFEP. Besides, one can consider decreased initial rate as another confirmation on the formation of soluble polyelectrolyte as it was confirmed by conductimetry.

\section{Effect of NaAA as comonomer with different concentration}

Table 3 shows different characteristics of latexes synthesized with various amounts of $\mathrm{NaAA}$, while other polymerization parameters were kept constant. Figure 6 shows their corresponding SEM images.

Interestingly, in direct contrast with $\mathrm{NaSS}$ and NaAmps cases, the addition of $\mathrm{NaAA}$ led to an increment in the average particle size. Additionally, the early-stage polymerization rate continuously declines as NaAA mole fraction increased.

Domination of the ionic strength over comonomer concentration and increasing critical chain length for precipitation are responsible for particle size increment.

Regarding the predomination of the ionic strength over comonomer concentration, an intriguing question can be posed: why this did not happen for sulfonic-based comonomers? This

Table 2 Effect of NaAmps mole percent on different characteristics of MBC-based samples

\begin{tabular}{|c|c|c|c|c|c|c|}
\hline Sample code & NaAmps in feed (mol\%) & particle size $(\mathrm{nm})$ & PDI & $\begin{array}{l}\text { Conversion at } \\
15 \mathrm{~min}\end{array}$ & $\begin{array}{l}\text { Conversion } \\
\text { at } 25 \mathrm{~min}\end{array}$ & $\begin{array}{l}\text { Final conversion } \\
\text { (after } 24 \text { h) }\end{array}$ \\
\hline $\mathrm{MBC0}$ & 0 & 350 & 0.009 & 15.2 & 23.7 & 98.5 \\
\hline MBC-NaAmps1.25 & 1.25 & 179 & 0.085 & 19.4 & 49.8 & 97.9 \\
\hline MBC-NaAmps 2.5 & 2.5 & 128 & 0.043 & 25.3 & 40.2 & 98.2 \\
\hline MBC-NaAmps5 & 5 & 112 & 0.078 & 17.1 & 29.7 & 98.6 \\
\hline MBC-NaAmps10 & 10 & - & - & 8.9 & 12.3 & 99.5 \\
\hline
\end{tabular}


Table 3 Effect of NaAA mole percent on different characteristics of MBC-based samples

\begin{tabular}{|c|c|c|c|c|c|c|}
\hline Sample code & $\mathrm{NaAA}(\mathrm{mol} \%)$ in feed & Particle size (nm) & PDI & Conversion at $15 \mathrm{~min}$ & Conversion at $25 \mathrm{~min}$ & Final conversion(after $24 \mathrm{~h}$ ) \\
\hline $\mathrm{MBC} 0$ & 0 & 350 & 0.009 & 15.2 & 23.7 & 98.5 \\
\hline MBC-NaAA1.25 & 1.25 & 372.5 & 0.182 & 14.2 & 19.3 & 96.9 \\
\hline MBC-NaAA2.5 & 2.5 & 391.9 & 0.097 & 12.5 & 15.8 & 98.1 \\
\hline MBC-NaAA5 & 5 & 613.8 & 0.016 & 9.8 & 12.7 & 99.2 \\
\hline MBC-NaAA10 & 10 & 804.4 & 0.046 & 6.2 & 10.5 & 97.6 \\
\hline
\end{tabular}

is due to higher strength of sulfonic acids based than carboxylic ones. For instance, $\mathrm{pKa}$ of sulfonic acid groups of poly(styrene sulfonate) is 1 [28], while this value for carboxylic groups of poly(acrylic acid) is 4.5 [29] (Note that these values are strongly dependent to polymer characteristics especially molecular weight); as a result, the influence of ionic strength on the several features of the carboxylic acids is higher than the sulfonic ones. From another point of view, their neutralizations respectively give strong and weak bases. It means that the salts of carboxylic acids based are more inclined to become protonated as compared to the sulfonic ones which is not in favor of stability and reducing particle size.

To clarify increasing chain length for precipitation, one can consider reactivity ratios which are $r_{\mathrm{MMA}}=0.7$ and $r_{\mathrm{NaAA}}=1.06$ for MMA/NaAA system [30]; thus, in the early stages of polymerization, relatively high amount of hydrophilic NaAA units will be polymerized; as a result, the nucleation stage would be delayed, which eventually leads to a lower particle number and higher particle size.

Using acrylic acid as comonomer and employing SFEP in water, Yan et al. [31] and Kang et al. [32] have reported a diverse trend of particle size variation. The addition of methanol to water as co-solvent reduces the dielectric constant of the medium from 80.1 to 62.5 [33]. As it was mentioned, this is not in favor of stabilization of newly formed nuclei; consequently, primary particles coagulate and the average particle size increases. On the other hand, particle size distribution has been dramatically broadened for the sample containing $1.25 \mathrm{~mol} \%$ of NaAA, which can be an indication of secondary nucleation. Afterward, increment of NaAA content to higher amounts results in narrower particle size distribution. Yamamoto [34] has described the narrowing size distribution of particles as a consequence of heterocoagulation between the primary and the secondary particles induced by increasing salt. Once again, it is noteworthy to point out the incorporation of comonomer is accompanied by increase in ionic strength.

Acrylic acid (without neutralization) resulted in latex destabilization. This can be attributed to the low acidic strength of acrylic acid $\left(\mathrm{pK}_{\mathrm{a}}=4.25\right)$ [35] together with low dielectric constant of the medium $(\varepsilon=62.5)$. In fact, both of these effects harden ionization ability of acrylic acid, leading to catastrophic coagulation. In the same manner, Guillaume et al. [36]
Fig. 6 SEM micrographs of MBC-based particles synthesized by SFEP at $75^{\circ} \mathrm{C}$ using water/ methanol $(65 / 35 \mathrm{w} / \mathrm{w})$ as the polymerization medium with various molar percentage of $\mathrm{NaAA}$ with respect to total monomers: 0 (a), 2.5 (b), 5 (c), and $10(\mathbf{d})$
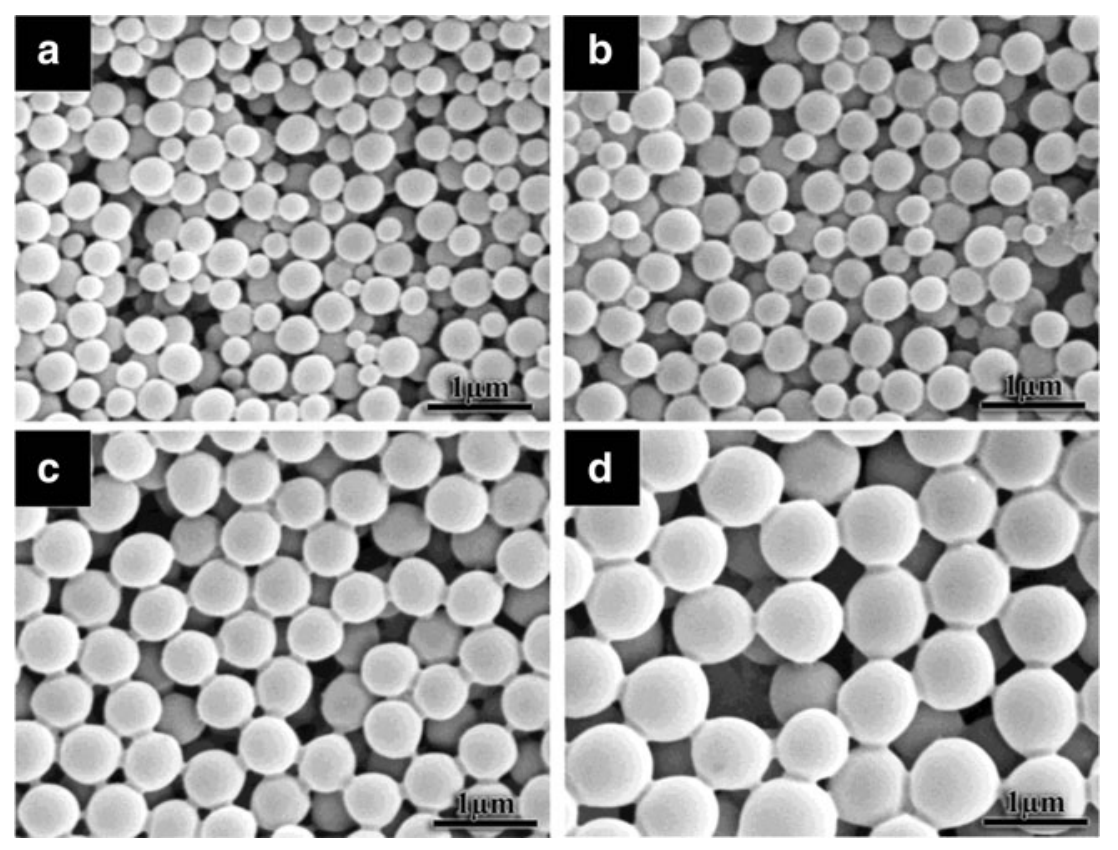
Table 4 Effect of $\mathrm{Na}_{2}$ ita mole percent on different characteristics of MBC-based samples

\begin{tabular}{|c|c|c|c|c|c|c|}
\hline Sample code & $\mathrm{Na}_{2}$ ita $(\mathrm{mol} \%)$ in feed & Particle size (nm) & PDI & Conversion at $15 \mathrm{~min}$ & Conversion at $25 \mathrm{~min}$ & Final conversion (after $24 \mathrm{~h}$ ) \\
\hline $\mathrm{MBC} 0$ & 0 & 350 & 0.009 & 15.2 & 23.7 & 98.5 \\
\hline MBC-Na ${ }_{2}$ ita 1.25 & 1.25 & 394 & 0.035 & 14.4 & 17.2 & 98.4 \\
\hline $\mathrm{MBC}-\mathrm{Na}_{2} \mathrm{ita} 2.5$ & 2.5 & 477 & 0.07 & 11.6 & 16.8 & 96.3 \\
\hline $\mathrm{MBC}-\mathrm{Na}_{2}$ ita5 & 5 & 677.2 & 0.005 & 8.2 & 11.3 & 95.1 \\
\hline MBC-Na ${ }_{2}$ ita10 & 10 & 862.4 & 0.118 & 7.1 & 9.8 & 95.9 \\
\hline
\end{tabular}

reported latex destabilization at $\mathrm{pH}<6.5$ in SFEP of styrene/ butyl acrylate using methacrylic acid as comonomer.

Effect of $\mathrm{Na}_{2}$ ita as comonomer with different concentration

Finally, $\mathrm{Na}_{2}$ Ita was used with different concentrations, while other polymerization parameters were kept constant. Table 4 and Fig. 7 respectively show characteristics of the latexes and their SEM images. Both the results and discussion are similar to NaAA case. However, as it can be seen, at a constant comonomer concentration, particle size is a little higher as compared to the NaAA case. All the samples were stable except for MBC-Na $\mathrm{Na}_{2} \mathrm{ita} 0$ which had about $12 \mathrm{wt} \%$ of coagulum.

Despite the fact that itaconic acid and their salts have a low reactivity in both solution homopolymerization [37] and emulsion copolymerization [38], Lock et al. [39] demonstrated that the addition of $\mathrm{Na}_{2}$ Ita to the copolymerization of MMA/BuA increases the water solubility of resulting polymer, which postpones the precipitation of terpolymer chains and eventually increases the average particle size in emulsion polymerization. Our results further verify inefficiency of $\mathrm{Na}_{2}$ Ita in lowering particle size and stability to such a degree that some coagulated particles were seen as the concentration of $\mathrm{Na}_{2}$ Ita increased to $10 \mathrm{~mol} \%$ (Fig. 7d). Furthermore, itaconic acid (without neutralization) was resulted in destabilization as it did for acrylic acid.

As can be seen in SEM micrographs, regardless of the size and distribution index of particles, the morphology of the particles is not perfectly spherical and in some point egg-like morphology is somewhat conspicuous, especially in MBCNaAA5 and MBC-NaAA10 samples (Figs. 6c, d and 7c). Kang et al. [40] were also obtained this kind of morphology in SFEP of methyl methacrylate, ethyl acrylate, and acrylic acid by alkali post-treatment.

\section{Conclusion}

Soap-free emulsion polymerization of methyl methacrylaye/ butyl acrylate was carried out in water/methanol mixture (65/ $35, w / w)$. Four different anionic comonomers, namely NaSS,
Fig. 7 SEM micrographs of MBC-based particles synthesized by SFEP at $75^{\circ} \mathrm{C}$ using water/ methanol $(65 / 35 w / w)$ as the polymerization medium with various molar percentage of $\mathrm{Na}_{2}$ ita with respect to total monomers: 0 (a), 2.5 (b), 5 (c), and $10(\mathbf{d})$
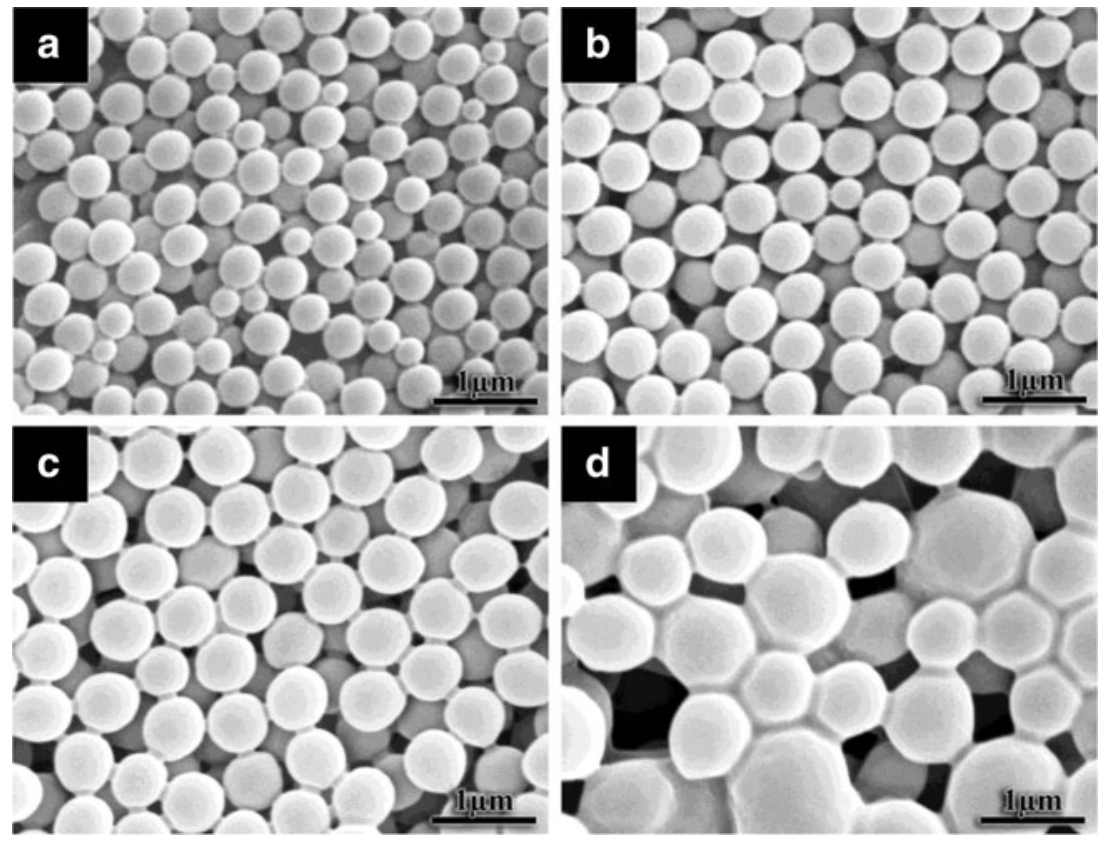
NaAmps, $\mathrm{NaAA}$ and $\mathrm{Na}_{2}$ Ita were used to functionalize, improve the stability and study their influence on the mean particle size and particle size distribution of the resulting MBC-based particles. The trends of soluble polyelectrolyte formation, particle size, colloidal stability, initial rate of the reaction were completely different in these cases. As the methanol content of the medium increased, the amount of soluble polyelectrolyte dramatically decreased in the case of carboxylic functionalized particles ( $\mathrm{NaAA}$ and $\mathrm{Na}_{2} \mathrm{Ita}$ ), while no obvious change was observed in the case of sulfonic-based particles (NaSS and NaAmps). Sulfonic-based comonomers first significantly reduced particle size, and then resulted in predomination of solution polymerization over SFEP. On the contrary, carboxylic ones increased particle size and at higher amount led to coagulation (especially in the case of $\mathrm{Na}_{2} \mathrm{ita}$ ). In addition, the onset comonomer in which soluble chains are formed, determined by conductimetry results, in the case of NaAmps was higher than NaSS. The obtained particle size results were in good qualitative accordance with the classical Smith-Ewart theory. In sum, it was found that the efficiency of these comonomers both in reducing particle size and stability is in this order: $\mathrm{NaSS}>\mathrm{NaAmps}>\mathrm{NaAA}>\mathrm{Na}_{2}$ ita.

Acknowledgment Our special thanks are due to Ferdos Taleb for her kind patience to read this work and providing us with her valuable remarks. Besides, the first author is immeasurably grateful to Adel Mosaic Company for their generously financial support.

Open Access This article is distributed under the terms of the Creative Commons Attribution License which permits any use, distribution, and reproduction in any medium, provided the original author(s) and the source are credited.

\section{References}

1. Warson H, Finch CA (2001) Applications of synthetic resin latices. Latices in diverse applications, vol 3. Wiley, Chichester

2. Kietzke T, Neher D, Landfester K, Montenegro R, Guntner R, Scherf U (2003) Nat Mater 2:408-412

3. Gao J, Yang Y, Lee D, Holdcroft S, Frisken BJ (2006) Macromolecules 39:8060-8066

4. Jong WHD, Borm PJA (2008) Int J Nanomedicine 3(2):133-149

5. Ma JZ, Hu J, Zhang ZJ (2007) Eur Polym J 43:4169-4177

6. Wilkinson MC, Hearn J, Steward PA (1991) Adv Colloid Interface Sci 81:77-165

7. Ceska GW (1974) J Appl Polym Sci 18:427-437
8. Shi M, Juang D, Krienger IM (1976) J Polym Sci Part A Polym Chem 14:2089-2107

9. Zhang F, Bai Y, Ma Y, Yang W (2009) J Colloid Interface Sci 33(4):13-21

10. Liu Z, Xiao H (2000) Polymer 41:7023-7031

11. Liu G, Liu P (2010) Colloid Surf A 354:377-381

12. Moribe H, Kitayama Y, Suzuki T, Okubo M (2012) Polymer J 44:205-210

13. Xu J, Li P, Wu C (1999) J Polym Sci Part A Polym Chem 37:20692074

14. Aymonier A, Papon E, Villenave J, Tordjeman P, Pirri R, Ge'rard P (2001) Chem Mater 13:2562-2566

15. Chonde Y, Krienger IM (1981) J Appl Polym Sci 26:1819-1827

16. An Z, Tang W, Hawker CJ, Stucky GD (2006) J Am Chem Soc 128:15054-15055

17. Ou JL, Yang JK, Chen H (2001) Eur Polym J 37:789-799

18. Adelnia H, Riazi H, Saadat Y, Hosseinzadeh S (2013) Colloid Polym Sci. doi:10.1007/s00396-013-2909-x

19. Saadat Y, Hosseinzadeh S, Afshar-Taromi F, Eslami H, Abdolbaghi $S$ (2012) Colloid Polym Sci. doi:10.1007/s00396-012-2812-x

20. Belleney J, Elary GH, Migonney V (2002) Eur Polym J 38:439-444

21. Bard AJ, Faulkner LR (1980) Electrochemical Methods: Fundamentals and Applications. Wiley, NewYork

22. Landfester K, Bechthold N, Forster S, Antonietti M (1999) Macromol Rapid Commun 20:81-84

23. Ciszkowska M, Kotlyar I (1999) Anal Chem 71:5013-5017

24. Davydova OV, Zelikin AN, Kargov SI, Izumrudov VA (2001) Macromol Chem Phys 202:1361-1367

25. English RJ, Gulati HS (1997) Rheol J 41(2):427-444

26. Zhang Q, Schattling P, Theato P, Hoogenboom R (2012) Polym Chem 3:1418-1426

27. Smith WV, Ewart RHJ (1948) Chem Phys 16:592

28. Lewis SR, Datta S, Gui M, Coker EL, Huggins F, Daunert S, Bachas L, Bhattacharyya D (2012) Proc Natl Acad Sci USA. doi:10.1073/ pnas. 1101144108

29. Greenwald HL, Luskin LS (1980) In: Davidson RL (ed) Handbook of water-soluble gums and resins. McGraw-1E11, New York

30. Shih CC, Wu KH, Chang TC, Liu HK (2008) Polym Compos 29(1):37-44

31. Yan C, Cheng SH, Feng L (1999) J Polym Sci Part A Polym Chem 37:2649-2656

32. Kang K, Kan CY, Du Y, Liu D (2004) J Appl Polym Sci 92:433-438

33. Pujari BR, Barik B, Behera B (1986) Phys Chem Liq 36:105-112

34. Yamamoto T (2012) Colloid Polym Sci 290:1023-1031

35. Han H, Lee J, Hong J, Shim SE (2009) Macromol Res 17(7):469475

36. Guillaume JL, Pichot C, Guillot J (1998) J Polym Sci Part A Polym Chem 26:1937-1959

37. Nagai S, Yoshida K (1960) K Kogaku 17:748

38. Egusa S, Makuuchi K (1982) J Polym Sci, Part A: Polym Chem 20:863

39. Lock M, EL-Aasser MS, Klein A, Vanderhoff JW (1991) J Appl Polym Sci 42:1065-1072

40. Kang K, Kan CH, Du Y, Liu D (2004) Polym Adv Technol 15:676-682 